NOTICE WARNING CONCERNING COPYRIGHT RESTRICTIONS:

The copyright law of the United States (title 17, U.S. Code) governs the making of photocopies or other reproductions of copyrighted material. Any copying of this document without permission of its author may be prohibited by law. 
CMU-CS-86-177

\title{
Specifying Functional and Timing Behavior for Real-Time Applications
}

\author{
M.R. Barbacci and J.M. Wing \\ Carnegie Mellon University \\ 26 November 1986
}

\begin{abstract}
We present a notation and a methodology for specifying the functional and timing behavior of real-time applications for a heterogeneous machine. In our methodology we build upon well-detined, though isolated, pieces of previous work: Larch and Real Time Logic. In our notation, we strive to keep separate the functional specification from the timing specification so that a task's functionality can be understood independent of its timing behavior. We show that while there is a clean separation of concerns between these two specifications, the semantics of both pieces as well as their combination are simple.

Comments, suggestions, criticisms, etc., are appreciated. Address them to:

Dr. Mario R. Barbacci

Software Engineering Institute

Carnegie Mellon University

Pittsburgh, PA 15213

(412) 268-7704

Barbacci@sei.cmu.edu.arpa

\author{
Professor Jeannette M. Wing \\ Department of Computer Science \\ Carnegie Mellon University \\ Pittsburgh, PA 15213 \\ (412) 268-3068 \\ Wing@c.cs.cmu.edu.arpa
}

This research is carried out jointly by the Software Engineering Institute, a Federally Funded Research and Development Center, sponsored by the Department of Defense, and by the Department of Computer Science, sponsored by the Defense Advanced Research Projects Agency (DOD), ARPA Order No. 4976. monitored by the Air Force Avionics Laboratory Under Contract F33615-84-K-1520. Additional support for J.M. Wing was provided in part by the National Science Foundation under grant DMC-8519254.

The views and conclusions contained in this document are those of the authors and should not be interpreted as representing official policies, either expressed or implied, of the Software Engineening Institute, Carnegie-Mellon University, the National Science Foundation, the Department of Defense or the US Government.
\end{abstract}




\section{Table of Contents}

1 Problem Context

2 Contributions

3 Introduction to Larch

4 Behavioral Information

4.1 Functional Specifications

4.1.1 Symtax and Meaning

4.1.2 Example

4.2 Timing Specifications

4.2.1 Syntax and Meaning

4.2.2 Example

5 Formal Meaning of Functional and Timing Specifications

5.1 Assigning Meaning to Timing Specifications

5.2 Assigning Meaning to the Combined Specifications

6 Examples

7 Related Work

8 Summary 


\section{Problem Context}

Many computation-intensive, real-time applications require efficient concurrent execution of multiple tasks devoted to specific pieces of the application. Typical tasks inctude sensor data collection, obstacle recognition, and global path planning, in applications such as robotics and vehicular control. Since the speed and throughput required of each task may vary, these applications can best exploit a computing environment consisting of multiple special and general purpose processors that are logically, though not necessarily physically, loosely connected. We call this environment a heterogeneous machine.

During execution time, processes, which are instances of tasks, run on possibly separate processors, and communicate with each other by sending messages of different types. Since the patterns of communication can vary over time, and the speed of the individual processors can vary over a wide range, additional hardware resources, in the form of switching networks and data buffers are required in the physical heterogeneous machine. Logically, queues are used to buffer data; processes dequeue data on queues attached to input ports and enqueue data from queves attached to output ports.

The application developer is responsible for prescribing a way to manage all of these resources. We call this prescription a task-level application description. It describes the tasks to be executed, the assignment of processes to processors, the data paths between the processors, and the intermediate queues required to store the data as it moves from source to destination processes. A task-level description language is a notation in which to write these application descriptions.

We are using the term "description language" rather than "programming language" to emphasize that a task-level application description is not translated into object code in some kind of executable "machine language." Rather, it is to be understood as a description of the structure and behavior of a logical machine, that will be synthesized into resource allocation and scheduling directives. These directives are to be interpreted by a combination of software, firmware, and hardware in a heterogeneous machine.

We have an initial design of such a description language [1], a compiler for it, and a simulator that takes task descriptions as input. A task description (see Figure 1) contains information about four aspects of a task: (1) its interface to other tasks (ports) and to the scheduler (signals); (2) its functional and timing behavior, (3) its attributes, and (4) its internal structure, thereby allowing for hierarchical task descriptions. Reference [1] contains a more complete explanation of these and other features of the language. In this paper we focus on only one aspect: the information appearing in the behavior part of a task description.

\section{Contributions}

Formal specifications have been used successfully for specifying the functional behavior of software systems, e.g., individual program modules and abstract data types. These specifications have traditionally been used to verify a program's correctness ("is the right answer computed?"). Often, however, one is interested in not only the functional correctness of a system but also other properties, such as reliability, performance, security, and real-time behavior. Less work has focused on formally specifying these other properties of software systems, let alone their interactions with each other.

To our knowledge no work has addressed the formal integration of the formal specification of functional and timing behavior of sottware. The main contribution of this paper is exactly this integration of functional and timing specifications as embodied in our task description language. 


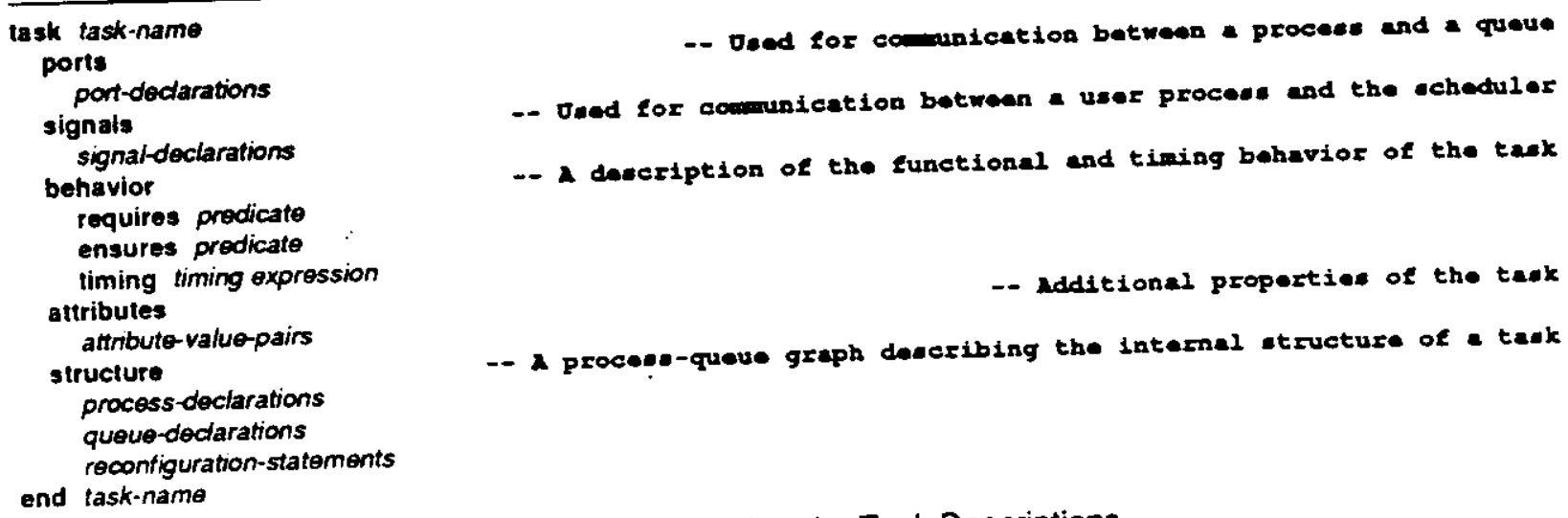

Figure 1: A Template for Task Descriptions

We combine two separate formalisms: an axiomatic specification language, Larch [11, 12], used to specity functional behavior, and an event expression language used to specity timing behavior. Both are mapped to the same underlying logic, typed first-order predicate logic, so that their combination has a formal semantics.

Two significant aspects of our work are as follows:

- Since the formal semantics is relatively simple (first-order logic), not only can people easily understand our specifications but the specifications themselves can easily be subject to machine analysis.

- We build upon previous well defined and isolated pieces of research and combine them in a meaningtul way. Their combination is applied in a context (heterogeneous machines) that itself is of growing interest to those involved in parallel architectures and languages.

\section{Introduction to Larch}

Before we describe the functional and timing specifications of a task, we give a brief introduction to Larch'.

Larch uses a two-tiered approach to specifying program modules: a trait defines state-independent properties, and an interface specification defines state-dependent properties of a program. A trait is written in the Larch Shared Language (LSL), and it provides the assertion language used to express and define the meaning of the predicates of an interface specification.

For a program module, such as a procedure, a Larch interface specification is written in a Larch Interface Language (LIL) and contains predicates about the states before and after the execution of the procedure. The Larch Interface Language to be used is specific to the programming language in which the procedure is written (e.g., C, Commonlisp. Ada, etc.). For this paper we will use a relatively simple interface language, such as would be defined for an Algol-like language.

\footnotetext{
'We are keeping this introduction to Larch very short. The reader is encouraged to consult the appropriate references in the bibliography.
} 


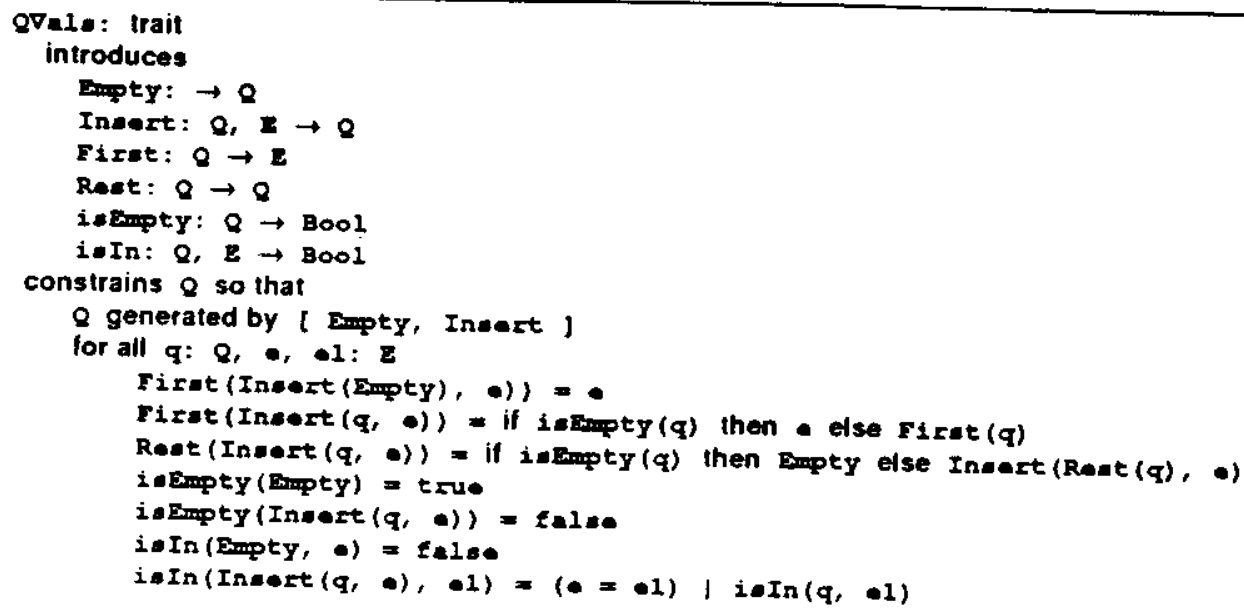

a. A Trait for Queue Values

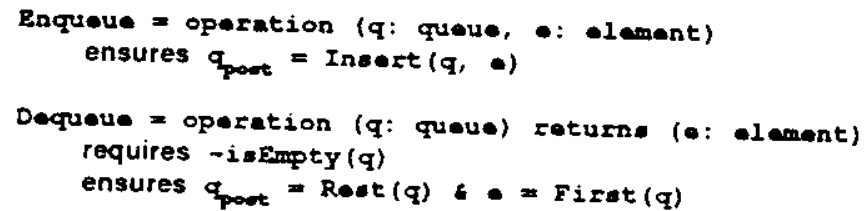

b. Interfaces for Queue Operations

Figure 2: A Larch Two-Tiered Specification for Queues

Figure 2 depicts a Larch (two-tiered) specification of queues with Enqueue and Dequeue operations. The top part of the specification (Figure 2.a) is a trait written in LSL used to describe values of queues. A trait is akin to an algebraic specification (see Section 7 on Related Work). A set of operators and their signatures following introduces defines a vocabulary of terms to denote values of a type. For example, Empty and Insert(Empty, 5) denote two different queue values. The set of equations following the constrains clause defines a meaning for the terms; more precisely, an equivalence relation on the terms, and hence on the values they denote. For example, from the above trait, one could prove that First $($ Rest (Insert(Insert(Empty, 5), 6))) $=6$.

The bottom part of the specification (Figure 2.b) contains two interfaces written in our "generic" Larch interface language. They describe the functional behavior of two queue operations, Enqueue and Dequeue (queue operation names are used to write timing expressions, which are described later in this paper). A requires is a pre-condition on the state of an operation's input data that must be true upon operation invocation; an ensures is a post-condition on the state of an operation's input and output data that is guaranteed to be true upon operation termination. An omitted predicate is taken to be true. The specification for Dequeue states that Dequeue must be called with a non-empty queue and that it modifies the original queue by removing its first element and retuming it. 


\section{Behavioral information}

The behavioral information in a task description is divided into two parts: a functional specification and a timing specitication. In the next two subsections we describe informally the syntax and meaning of these two specifications. Section 5 gives the formal meaning, and in particular, the meaning of the combination of functional and timing specifications.

\subsection{Functional Specifications}

\subsubsection{Syntax and Meaning}

The functional information of a task description (see Figure 1) describes the behavior of the task in terms of predicates about the data in the queues, before and after each execution of the task. It consists of a requires clause and an ensures clause, together constituting a simple Larch interface specification. LSL is used as the assertion language in the predicates of these clauses.

A requires clause states what is required to be true of the data coming through the input ports; an ensures clause states what is guaranteed to be true of the data going out through the output ports. If one were to view each cycle of a task as one execution of a procedure, the requires and ensures are exactly the pre- and post-conditions on the functionality of that cycle.

A task implementation must satisty the predicates, $R$ and $E$, of the requires and ensures clauses. $A$ task implementation is simply a program written in some programming language, e.g., C, CommonLisp, or Ada. Using Hoare-like notation, an implementation, Prog, satisfies the (functional) specification if:

\section{\{R\} Prog $\{$ E\}}

It is up to the task implementor to show that a task implementation satisfies the functional specification as given by the requires and ensures clauses. This verification can be done formally - standard verification techniques can be used $([13,14])$ and some mechanical tools are available to aid this process $([9,19,22,21])$. We defer to Section 5.2 for the definition of the meaning of the predicates in the presence of timing information.

\subsubsection{Example}

Consider a matrix multiplication task (Figure 3) that takes input matrices from two queues and outputs the result matrix on an output queue. The data traveling through these ports are of type matrix. Matrix values are specified using LSL just as for queue values, so "rows," "cols" and ".". would be defined in a trait about matrix values. The requires clause states that the task implementor may assume that the number of rows of the matrix entering through the port in1, equals the number of columns of the matrix entering through in 2 . The ensures clause states that the result of multiplying the two input matrices is output through the output port.

\subsection{Timing Specifications}

\subsubsection{Syntax and Meaning}

The timing information describes the behavior of the task in terms of the operations that it performs on the queues attached to its input and output ports; this is the behavior of the task seen from the outside. 


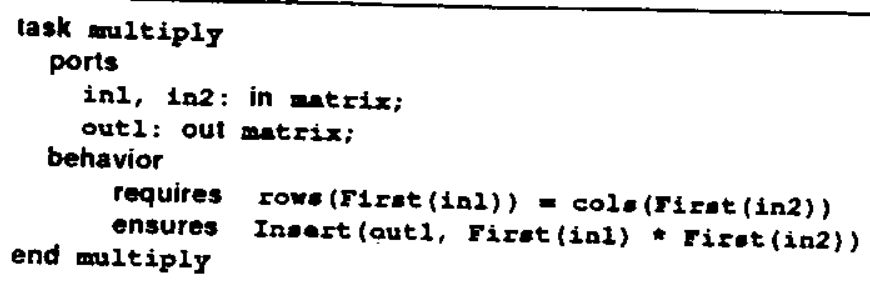

Figure 3: The Functionality of a Matrix Multiplication Task

The simplest timing expression is the name of a queve operation, e.g., Enqueue or Dequeue, on a queue attached to a specific port, e.g., int. The duration of a queue operation or the delay between two operations is described by a time window. Time windows are denoted by a pair of time values $\left[T_{\min }, T_{\max }\right]$ defining the boundaries of the interval. The time window associated with a queue operation describes the minimum and maximum time needed to perform the operation. Intervals of time between queue operations are denoted by a Delay "operation" whose time window describes the minimum and maximum time consumed by the process in between queue operations.

A composite timing expression denotes the sequential and/or concurrent execution of operations on queues. Sequential composition is denoted by a space between operations; parallel composition is denoted by a "II" between operations. For example,

loop (in1.Dequeue[10,15] || in2.Dequeue) delay[",30] out1.Enqueue

is a sequential timing expression that specifies two parallel Dequeue operations on the queues attached to the input ports in1 and in2 followed, after some delay, by an Enqueue on the queue attached to the output port out1. The Delay lasts some undetermined amount of time less than 30 seconds. The Dequeue operation on port in 1 takes between 10 and 15 seconds to complete. The other two operations take some implementation dependent default time to complete. The keyword loop denotes a cyclic or repeating task.

An optional guard in a timing expression specifies:

1. the number of times the task is to be executed: "repeat integer $\Rightarrow>$ expression," or

2. during what time interval the task is allowed to start: "during timewindow => expression," or

3. the earliest allowable start time: "after timevalue => expression," or

4. the latest allowable start time "before timevalue => expression," or

5. a predicate on the state of the input queues or the current time which must be true before the task is allowed to start: "when predicate => expression."

In our examples, we will often drop the name of the queue operation and use just the name of the port (i.e., "in 1" instead of "in 1.Dequeue"). Since this paper introduces only two queue operations: Enqueue and Dequeue, and given that the former applies only to input queues and the other applies only to output queues, no confusion should occur as to which operation is implied. 


\subsubsection{Example}

Consider a matrix multiplication task (Figure 4) that takes input matrices from two queues and outputs the result matrix on an output queue. The timing ctause states that the task does not start executing until both input queues contain data. Once that condition is satisfied, the task will remove its input data from both input queues concurrently (the Dequeue operations), will operate on the data for between 10 and 15 seconds (this "computation" time is lumped together under the delay operation), and finally will enqueue some output in the output queue. Notice another use of LSL in our specifications: the when condition places a constraint on the state of the queues (not on the state of the data in the queves). We use the trait from Section 3 to define the assertion language for predicates in a when guard.

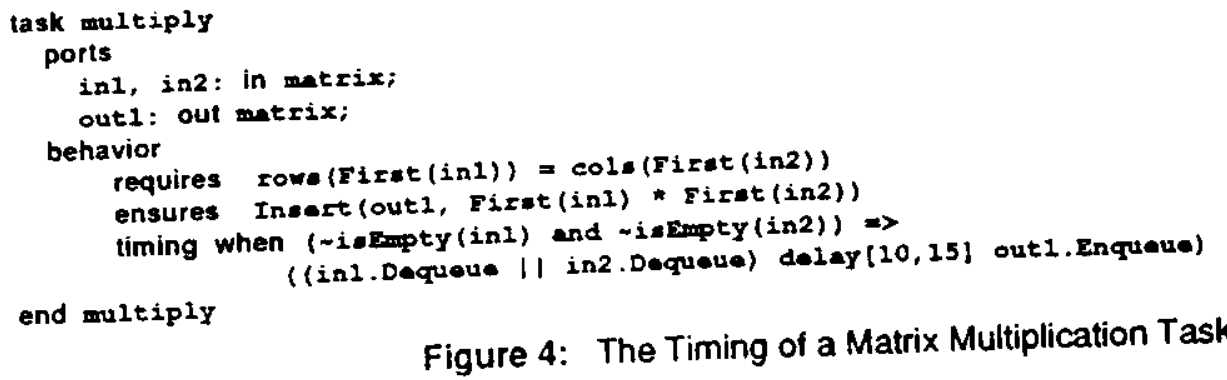

Figure 4: The Timing of a Matrix Multiplication Task

5 Formal Meaning of Functional and Timing Specifications

We use Jahanian and Mok's Real-Time Logic (RTL) [15] to give meaning to our timing expressions. Furthermore, we use their logic to give meaning to the combination of our functional and timing specifications. We use four of their notational conventions:

Syntax Meaning

$\uparrow A$

The start of an operation ("action" in RTL's terminology).

$\downarrow A \quad T$ The end of an operation.

$@(E, i) \quad$ The time of the $i^{\text {th }}$ occurrence of event $E$, where events in our context are the start of an operation or the end of an operation. @ is an occurrence function that captures the notion of real-time.

The interval of time during which the predicate $P$ holds. $P$ holds before or at 11 , from $\mathrm{t} 1$ to $\mathrm{t} 2$, and at or after $\mathrm{t} 2$. If $\mathrm{t} 1$ and $\mathrm{t} 2$ are identical, then $\mathrm{P}$ holds at an interval around t1. For brevity, we will use $P(t)$ when $t 1=t 2$ (i.e., " $P$ holds around time $t$ ").

5.1 Assigning Meaning to Timing Specifications

In this section we describe the meaning of our timing specifications in terms of RTL logic. In the following discussion, we assume E, E1, and E2 are arbitrary timing expressions; $A, A 1$, and $A 2$ are operations; $t 1$ and $t 2$ are times (absolute or relative); $a 1$ and $a 2$ are absolute times; $r 1$ and $r 2$ are relative times; and $W$ is a predicate of a when guard.

To simplity the exposition, we introduce a simple rewrite rule: Any timing expression of the form "repeat $n \Rightarrow E^{\prime}$ can be rewritten as a sequence of $n$ occurrences of the unguarded expression $E$ ("E EE ... $E$ ").

Thus, the only guards we need to consider are before. after, during, and when. 
We also introduce the following axioms:

1. For any queve operation $A$, and for some implementation defined duration $T$, the following axiom expresses the duration of $A$ :

$$
\forall i[@(\downarrow A, i)-@(\uparrow A, i)=T]
$$

2. For any queue operation A[t1, t2], with a duration defined by the time window [t1,t2], the following axiorn expresses the duration of $A$ :

$$
\forall i[11 \leq @(\downarrow A, i)-@(\uparrow A, i) \leq t 2]
$$

3. For any sequence of queue operations, $A 1$... An, the following axiom relates the start and end times of the sequence to the start and end times of the individual operations:

$$
\forall i[@(\uparrow A, i)=@(\uparrow A 1, i) \wedge @(\downarrow A, i)=@(\downarrow A n, i)]
$$

4. For any parallel queve operations, A1 $\|\ldots\|$ An, the following axiom relates the start and end times of the composition to the start and end times of the individual operations:

$$
\forall i[@(\uparrow A, i)=\min (@(\uparrow A 1, i), \ldots . . @(\uparrow A n, i)) \wedge @(\downarrow A, i)=\max (@(\downarrow A 1, i), \ldots, @(\downarrow A n, i))]
$$

5. The last two axioms state that cycles in a repeating task do not overlap. Thus, we cannot have an input operation finish after any of the output operations and we cannot have an output operation start before any input operation starts:

$$
\begin{aligned}
& \forall i\left[\max \left(@\left(\downarrow_{\text {out }}, i\right), @\left(\downarrow_{\text {out }}, i\right), \ldots, @\left(\downarrow_{\text {out }}, i\right)\right)>\max \left(@\left(\operatorname{Lin}_{1}, i\right), @\left(\downarrow_{\text {in }}, i\right), \ldots, @\left(\downarrow_{\text {in }}, i\right)\right)\right] \\
& \forall i\left[\min \left(@\left(\text { out }_{1}, i\right), @\left(\text { Tout }_{2}, i\right), \ldots, @\left(\text { out }_{\mathrm{J}}, \mathrm{i}\right)\right)>\min \left(@\left(\uparrow_{\mathrm{in}}, \mathrm{i}\right), @\left(\uparrow_{\mathrm{in}}, \mathrm{i}\right), \ldots, @\left(\text { in }_{\mathrm{K}}, \mathrm{i}\right)\right)\right]
\end{aligned}
$$

where $J$ and $K$ are the number of output and input queues, respectively.

We assign a meaning to timing expressions by introducing a function, $M_{4}$ (Table 1.a), which maps timing expressions to Boolean values.

$$
\mathrm{M}_{\mathrm{t}} \text { : Timing Expression } \rightarrow \text { Boolean. }
$$

We use an auxiliary function, op (Table 1.b), which maps timing expressions to operations, op: Timing Expression $\rightarrow$ Operation.

$o p$ is needed because "start time" and "end time" are meaningful only for queue operations.

As an example of how to interpret the formalism intuitively, consider the entries for the during guard in Table 1.a. They specify a time window during which the operation is allowed to start. The first value is the earliest start time allowed and must be an absolute time value. The second value is the latest start time allowed and can be an absolute time value or a time value relative to the former. The meaning of the guarded expression is the conjunction of the meaning of the expression proper and a predicate stating the restriction on starting times.

\subsection{Assigning Meaning to the Combined Specifications}

Given a task description of the form:

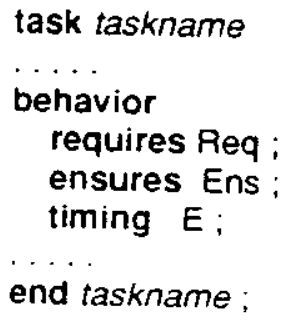




\section{Timing Expression}

E1 ... En

E1\|...\|En

E1 E2

\section{E1 \| E2}

when $W \Rightarrow E 1$

before a1 $=>E 1$

after $a 1 \Rightarrow E 1$

during $[a 1, a 2] \Rightarrow E 1$

during $[a 1, r 2] \Rightarrow E_{1}$

$A[r 1, r 2]$

$A[*, r 1]$

$A[r 1, "]$

A

Timing Expression

loop E1

E1 ... En

E1 $\|\ldots\| \mathrm{En}$

$G \Rightarrow E_{1}$

$A[t 1, t 2]$

\section{$\underline{M_{4}}$ (Expression)}

$M_{1}(E 1)$

$M_{4}((E 1 E 2)$...En)

$\wedge M_{t}\left(E_{i} \|\left(E_{j}\right)\right.$ for all $i \neq i$

$M_{4}(E 1) \wedge M_{4}(E 2) \wedge \forall i[@(\downarrow o p(E 1), i) \leq @(\uparrow o p(E 2), i)]$

$M_{4}(E 1) \wedge M_{4}(E 2) \wedge$

$\forall i[@(\uparrow o p(E 1), i)<@(\downarrow o p(E 2), i) \wedge @(\uparrow o p(E 2), i)<@(\downarrow o p(E 2), i)]$

$M_{t}(E 1) \wedge \forall i[W(@(\uparrow o p(E 1), i))]$

$M_{4}(E 1) \wedge \forall i[@(T o p(E 1), i 1) \leq a 1]$

$M_{4}(E 1) \wedge \forall i[@(\uparrow o p(E 1), i) \geq a 1]$

$\mathrm{M}_{\mathrm{t}}(\mathrm{E1}) \wedge \forall \mathrm{i}[\mathrm{a} 1 \leq @(\uparrow o p(E 1), \mathrm{i}) \leq \mathrm{a} 2]$

$\mathrm{M}_{\mathrm{f}}(\mathrm{E} 1) \wedge \forall \mathrm{i}[\mathrm{a} 1 \leq @(\uparrow o p(E 1), \mathrm{i}) \leq \mathrm{a} 1+\mathrm{R}]$

$\forall i[@(\uparrow A, i)+r i \leq @(\downarrow A, i) \leq @(\uparrow A, i)+r 2]$

$\forall i[@(\downarrow A, i) \leq @(\uparrow A, i)+r 1]$

$\forall i[@(\uparrow A, i)+r 1 \leq @(\downarrow A, i)]$

true

a. Mapping from Timing Expressions to Booleans

op(Expression)

$o p(E 1)$

$o p(E 1) \ldots o p(E n)$

$o p(E 1)\|\ldots\|$ op(En)

$o p(E 1)$ for all guards $G$ (when, before, during, and after).

A

A

b. Mapping From Timing Expressions to Operations

Table 1: Assigning Meaning to Timing Expressions 
we give meaning to the predicates of the functional specification as related to time (i.e., at what times are these predicates to hold?) via a function $\mathbf{M}_{f}$ which maps from behavioral specifications to Boolean values:

$M_{4}$ : Predicate $\times$ Timing Expression $\rightarrow$ Boolean

\section{Predicate}

Req

Ens

\section{Timing Expression}

E
Mi(Predicate, Expression)

$\forall i\left[\operatorname{Req}(@(\uparrow o p(E), i)) \wedge M_{q}(E)\right]$ $\forall i\left[E n s(@(\downarrow o p(E), i)) \wedge M_{t}(E)\right]$

The function $\mathrm{M}_{4}$ is precisely the link between the functional and timing specifications. This link is characterizable purely in terms of first-order logic.

\section{Examples}

Figure 5 shows our multiply task with functional and timing information together. The figure shows two different multiply tasks, specified to have the same functionality but with different timing behavior. The timing expression in Figure 5.a states that the multiply task first checks that the input queues are nonempty, and if so perform two parallel Dequeue operations followed by an Enqueue operation. The timing expression in Figure 5.b states that the inputs come in sequentially instead of in parallel.

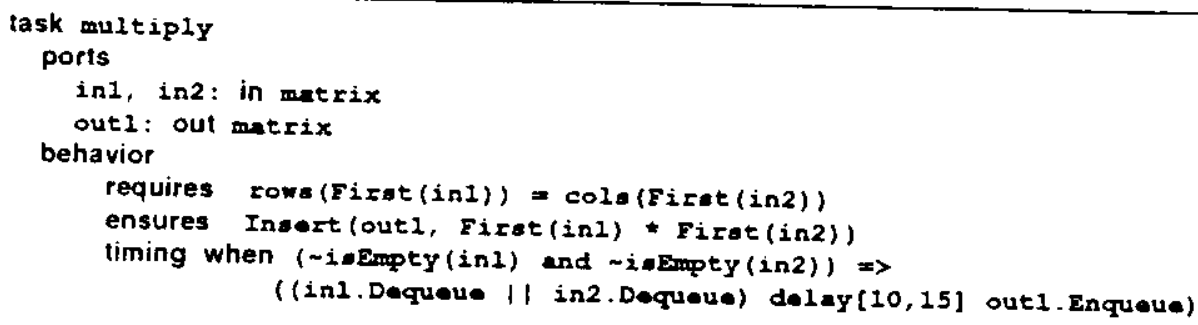

a. Parallel Input

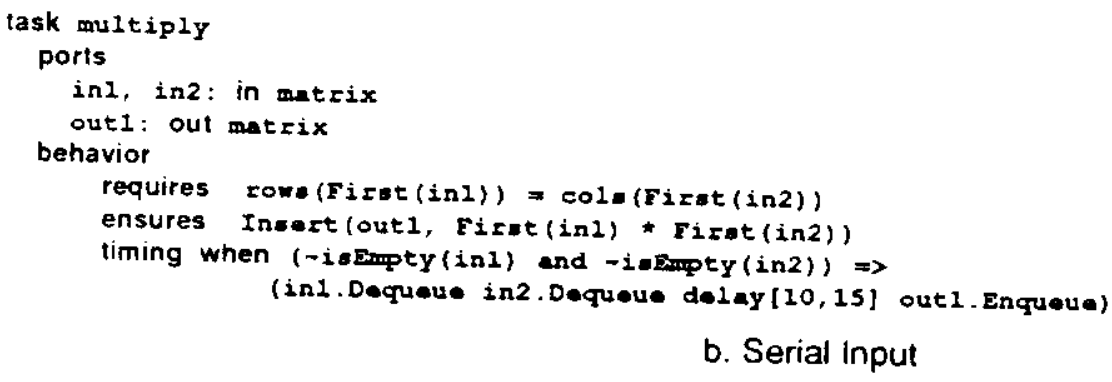

b. Serial input

Figure 5: Matrix Multiplication Task

To further illustrate the richness of our specification language and to show the benefits of cleanly separating the functional from the timing information, we write three alternative descriptions for a task built into our library. This task, deal, has one input port and a number of output ports. Data dequeued from the input port is enqueued to one of the output ports, but this can be implemented in a number of ways, as illustrated in Figure 6, below ${ }^{2}$.

\footnotetext{
${ }^{2}$ Assume that second(in 1), third(in 1), and fourth(in1) as abbreviations for First (Rest(int)), First(Rest(Rest(in1))), First(Rest( Rest(Rest(int)) ), respertively, are defined in the trat for queues.
} 
The first example (Figure 6.a) states that we altemate the dequeueing of input and enqueueing of output and ensures that first (second) output queue will see the first (second) item removed from the input queue. The second example (Figure 6.b) states that we dequeue all input before the output operations start, which themselves take place concurrently. It allows for the first dequeued data item to be enqueved on either of the output queues, but ensures that the second dequeued item will not be enqueued to the same as the first. The third example (Figure 6.c) states that input data are dequeued and grouped in pairs before enqueueing them into the output ports. The first pair is enqueued to the first output queue; the second pair, to the second.

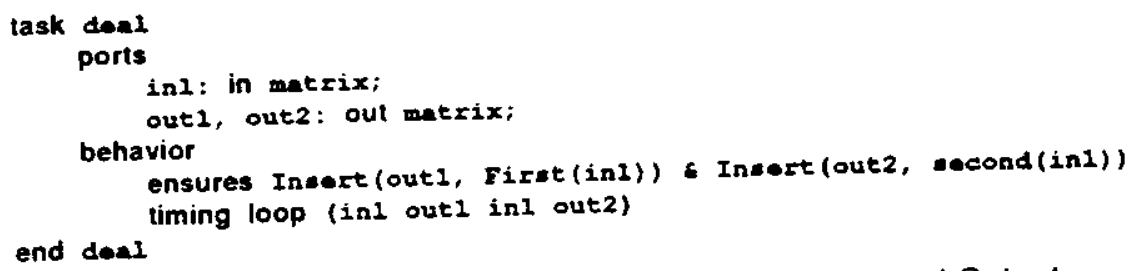

a. Alternating Input and Output

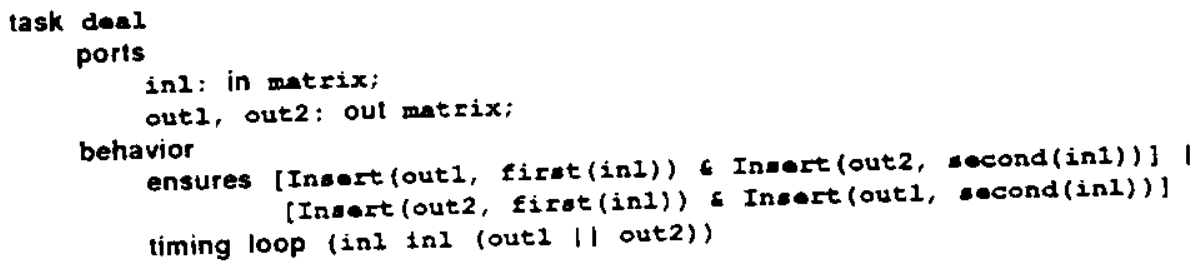

b. Concurrent Output

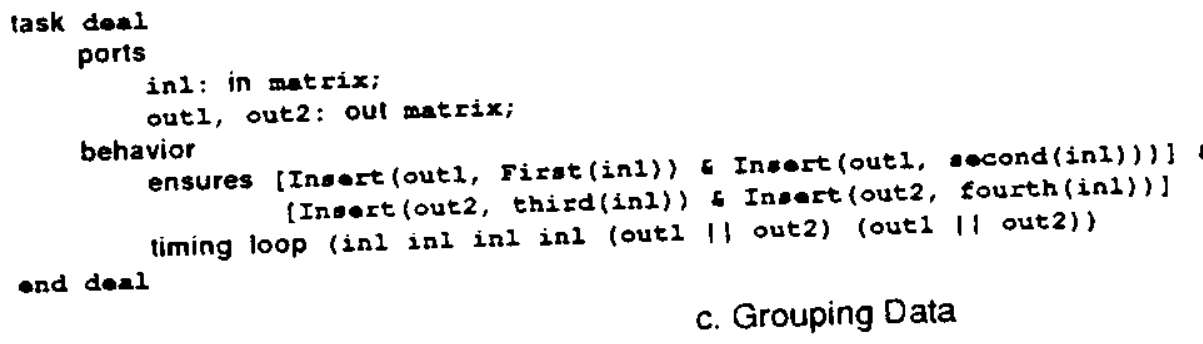

c. Grouping Data

Figure 6: Deal Task

\section{Related Work}

The axiomatic approach to specifying a program's functional behavior has its origins in Hoare's early work on verification [13] and later work on proofs of correctness of implementations of abstract data types [14]. where first-order predicate logic pre- and post-conditions are used for the specitication of each operation of the type. The algebraic approach, which defines data types to be heterogeneous algebras [2], uses axioms to specity properties of programs and abstract data types, but the axioms are restricted to equations. Much work has been done on algebraic specifications for abstract data types $[8,7,10,27,3,6,25,16]$; we use more recent work on Larch specifications [11, 12] for program modules. None of this work addresses the formal specification of timing behavior of systems. 
Operational approaches, such as those based on Timed Petri-net models [20, 23], are more commonly used for specitying behavior of real-time systems. Timed Petri-nets can be roughly characterized by whether "operation" time is assigned to the transitions, as in the original model by Ramchandani [20], or is assigned to the places, as in Sifakis' model [23]. In addition, both deterministic and stochastic timing are allowed, giving origin to a variety of models for specifying or evaluating performance requirements. This has been illustrated in recent work by Coolahan [4] (places, deterministic), Smith [24] (transitions, deterministic), Wong [26] (places, stochastic), and Zuberek [28] (transitions, stochastic). In contrast, our work takes a more axiomatic than operational approach to specifying timing behavior.

Specification and verification of timing requirements for real-time systems include recent work by Dasarthy [5], and by Lee, Gehlot, and Zwarico [17, 29]. This work as well as that by Jahanian and Mok, whose real-time logic we borrow, all focus on timing properties and not on functional behavior. Either states are left uninterpreted or predicates on states are simplistic, e.g., boolean modes as in Jahanian and Mok's work. In contrast, since we have a formal means of specifying the functional behavior of tasks and the data on which they operate, we have a more expressive specification language with a richer
semantics.

\section{Summary}

Our approach to specifying the functional and timing behavior of real-time applications for a heterogeneous machine has the following characteristics:

- It takes advantage of two well defined, though isolated, pieces of previous work.

- There is a clean separation of concerns between the two specifications.

- The semantics of both specifications as well as their combination are simple.

In our language design, we strove to separate the functional specification from the timing specification so that a task's functionality could be understood independent of its timing behavior. This separation of concerns gives us the usual advantages of modularity. Different timing specifications can be attached to the same functional specification. Task implementors can focus on satisfying functionality first, timing second. Task validation can be performed separately. For example, one could use formal verification for functionality and simulation for timing.

Since the semantics can be given in terms of first-order predicate logic, our specifications are amenable to machine manipulation and analysis. The algebraic style of Larch traits can be analyzed by rewrite-rule tools, e.g., Reve [18]; the two-state predicates of Larch interfaces and thus, task predicates, can be analyzed by verification systems that support first-order reasoning, e.g., Gypsy, HDM, and FDM $[9,21,22]$; formulae in real-time logic can be mechanically transformed into equivatent formulae in Presburger arithmetic. However, though many of these tools are available, much work is needed to integrate them so our specifications could be machine checked and analyzed. 


\section{References}

[1] M.R. Barbacci and J.M. Wing.

Durra: A Task-level Description Language.

Technical Report, Software Engineering Institute, Carnegie Mellon University, 1986.

G. Birkhoff and J.D. Lipson.

Heterogeneous Algebras.

Joumal of Combinatorial Theory 8:115-133, 1970.

[3] R.M. Burstall, and J.A. Goguen.

Putting Theories Together to Make Specifications. In Fifth International Joint Conference on Artificial Intelligence, pages 1045-1058. August, 1977. Invited paper.

[4] J.E. Coolahan and N. Roussopulos.

A Timed Petri Net Methodology for Specitying Real-Time System Requirements.

in International Workshop on Timed Petri Nets, pages 24-31. IEEE Computer Society Press, Torino, Italy, July, 1985.

[5] Dasarthy.

Timing Constraints of Real-Time Systems: Constructs for Expressing Them, Methods of Validating Them.

IEEE Transactions on Sottware Engineering 11(1):80-86, January, 1985.

[6] H.-D. Ehrich.

Extensions and Implementations of Abstract Data Type Specifications.

Lecture Notes in Computer Science. Volume 64. Proceedings of the 7th Symposium on Mathematical Foundations of Computer Science.

Springer-Verlag, 1978, pages 155-164.

[7] H. Ehrig and B. Mahr.

Fundamentals of Algebraic Specification 1.

Springer-Verlag, 1985.

[8] J.A. Goguen, J.W. Thatcher, E.G. Wagner, and J.B. Wright. Abstract Data Types as Initial Algebras and Correctness of Data Representations. In Proceedings from the Conference of Computer Graphics, Pattern Recognition and Data Structures, pages 89-93. ACM, May, 1975.

[9] D.I. Good, R.M. Cohen, C.G. Hoch, L.W. Hunter, and D.F. Hare.

Report on the Language Gypsy, Version 2.0.

Technical Report ICSCA-CMP-10, Certifiable Minicomputer Project, The University of Texas at Austin, September, 1978.

[10] J.V. Guttag.

The Specification and Application to Programming of Abstract Data Types.

PhD thesis, University of Toronto, Toronto, Canada, September, 1975.

[11] J.V. Guttag, J.J. Horning, and J.M. Wing.

Larch in Five Easy Pieces.

Technical Report 5, DEC Systems Research Center, July, 1985.

[12] J.V. Guttag, J.J. Horning, and J.M. Wing.

The Larch Family of Specification Languages.

IEEE Software 2(5):24-36, September, 1985.

[13] C.A.R. Hoare.

An axiomatic basis for computer programming.

Communications of the ACM 12(10):576-583. October, 1969. 
[14] C.A.R. Hoare.

Proof of Correctness of Data Representations.

Acta Informatica 1(1):271-281, 1972.

[15] F. Jahanian and A.K. Mok.

Safety Analysis of Timing Properties in Real-Time Systems.

IEEE Transactions on Sottware Engineering 12(9):890-904, September, 1986.

[16] S. Kamin.

Final Data Types and Their Specification.

ACM Transactions on Programming Languages and Systems 5(1):97-121, January, 1983.

[17] I. Lee, and V. Gehlot.

Language Constructs for Distributed Real-Time Programming.

In Proceedings of the Real-Time Systems Symposium, pages 57-66. San Diego, December, 1985.

[18] P. Lescanne.

Computer Experiments with the REVE Term Rewriting System Generator.

In Proceedings of Tenth Symposium on Principles of Programming Languages, pages 99-108. ACM, Austin, Texas, January, 1983.

[19] D.R. Musser.

Abstract Data Type Specification in the Affirm Systern.

IEEE Transactions on Software Engineering 6(1):24-32, January, 1980.

[20] C. Ramchandani.

Analysis of Asynchronous Concurrent Systems by Petri Nets.

Technical Report TR-120, MIT Project MAC, 1974.

[21] L. Robinson, and O. Roubine.

SPECIAL - A Specification and Assertion Language.

Technical Report CSL-46, Stanford Research Institute, Menlo Park, Ca., January, 1977.

[22] J. Scheid and S. Anderson.

The Ina Jo Specification Language Reference Manual.

Technical Report TM-(L)-6021/001/00, System Development Corporation, Santa Monica, CA, March, 1985.

[23] J. Sifakis.

Use of Petri Nets for Performance Evaluation.

In Proceedings of the IFIP Third International Workshop on Modeling and Pertormance Evaluation of Computer Systems, pages 75-93. North-Holland Publishing Co., Amsterdam, The Netherlands, 1977.

[24] C.U. Smith.

Robust Models for the Performance Evaluation of Hardware/Software Designs.

In International Workshop on Timed Petri Nets, pages 172-180. IEEE Computer Society Press, Torino, Italy, July, 1985.

[25] M. Wand.

Final Algebra Semantics and Data Type Extensions.

Journal of Computer and System Sciences 19(1):27-44, August, 1979.

[26] C.Y.Wong, T.S. Dillon, and K.E. Forward.

Timed Places Petri Nets With Stochastic Representation of Place Time.

In International Workshop on Timed Petri Nets, pages 96-103. IEEE Computer Society Press,

Torino, italy, July, 1985. 
[27] S.N. Zilles. Abstract Specifications for Data Types.

IBM Research Laboratory, San Jose, CA, 1975.

[28] W.M. Zuberek.

Performance Evaluation Using Extended Timed Petri-nets.

In International. Workshop on Timed Petri Nets, pages 272-278. IEEE Computer Society Press, Torino, Italy, July, 1985.

[29] A. Zwarico and I. Lee. Proving a Network of Real-time Processes Correct. in Proceedings of Real-Time Systems Symposium, pages 169-177. San Diego, December, 1985. 